\title{
Nonlinear vibrations in homogeneous non-prismatic Timoshenko cantilevers
}

\author{
Navid Navadeh ${ }^{\mathrm{a}}$, Pooya Sareh ${ }^{\mathrm{b} *}$, Volodymyr Basovskyc ${ }^{\mathrm{c}}$ Irina Gorban ${ }^{\mathrm{c}}$, Arash S. Fallah ${ }^{\mathrm{a}, \mathrm{d}, \mathrm{e}}$ \\ ${ }^{a}$ City and Guilds Building, Department of Aeronautics, South Kensington Campus, Imperial College London, London SW7 \\ 2AZ, United Kingdom \\ ${ }^{\mathrm{b}}$ Creative Design Engineering Lab (Cdel), Department of Mechanical, Materials, and Aerospace Engineering, School of \\ Engineering, University of Liverpool, Liverpool, The Quadrangle, Brownlow Hill, L69 3GH, United Kingdom \\ c Institute of Hydromechanics, National Academy of Sciences of Ukraine, 8/4 Zhelyabova st., Kiev 03680, Ukraine \\ ${ }^{\mathrm{d}}$ Institute of Computational Physics, Zürich University of Applied Sciences (ZHAW), Winterthur 8400, Switzerland \\ ${ }^{e}$ Department of Mechanical, Electronic, and Chemical Engineering, OsloMet, Pilestredet 35, 0166 Oslo, Norway \\ *Corresponding author. Email: pooya.sareh@liverpool.ac.uk
}

\begin{abstract}
Deep cantilever beams, modelled using Timoshenko beam kinematics, have numerous applications in engineering. The present study deals with the nonlinear dynamic response in a non-prismatic Timoshenko beam characterized by considering the deformed configuration of the axis. The mathematical model is derived using the extended Hamilton's principle under the condition of finite deflections and angles of rotation. The discrete model of the beam motion is constructed based on the finite difference method (FDM), whose validity is examined by comparing the results for a special case with the corresponding data obtained by commercial finite element (FE) software ABAQUS 2019. The natural frequencies and vibration modes of the beam are computed. These results demonstrate decreasing eigenfrequency in the beam with increasing amplitudes of nonlinear oscillations. The numerical analyses of forced vibrations of the beam show that its points oscillate in different manners depending on their relative position along the beam. Points close to the free end of the beam are subject to almost harmonic oscillations, and the free end vibrates with a frequency equal to that of the external force. When a point approaches the clamped end of the beam, it oscillates in two-frequency mode and lags in phase from the oscillations of the free end. The analytical model allows for the study of the influence of each parameter on the eigenfrequency and the dynamic response. In all cases, a strong correlation exists between the results obtained by the analytical model and ABAQUS, nonetheless, the analytical model is computationally less expensive.
\end{abstract}

Keywords: non-prismatic Timoshenko beam; nonlinear oscillation; finite difference method; amplitude-dependent frequency; pulse loading. 


\section{Introduction}

The model of a non-prismatic beam is applied when a beam's cross-sectional dimensions vary along its length. Different beam theories have been used to study the static and dynamic behaviour of uniform and non-prismatic beams [1-3]. However, the most accurate theory, as long as the restrictive plane deformation of a cross-section is assumed, is that of the Timoshenko beam theory [4]. Ever since its inception, the Timoshenko beam theory has been used to study a variety of beam problems when aspect ratios are such that simpler theories are rendered inaccurate $[1,5,6]$. The two distinctive aspects of the Timoshenko beam theory are the inclusion of shear deformation and rotatory inertia terms which distinguish it from the Euler-Bernoulli beam theory. While shear deformation is observed in static and dynamic applications, rotatory inertia effects can only be appreciated in dynamic applications. Although Timoshenko's beam theory is a well-trodden area of research, there has been a recent resurgence of interest in this topic in relation to the study of beam vibrations. The reasons behind this are numerous, nonetheless, this is primarily due to requirements for certain applications for which this theory is most suitable.

Some recent works dealing with the vibration of a non-prismatic beam are based on Chebyshev's series approximation method [7]. It is also necessary to mention the study of beam vibrations on an inertial elastic half-plane [7], and the study of the buckling and vibration of nanowires with surface effects and multi-walled carbon nanotubes as applications in nanotechnology [8, 9]. Rotating uniform Timoshenko beams were also studied theoretically [10,11]. Furthermore, more recently, studies of rotating tapered Timoshenko beams with the help of the finite element method have been conducted [11-18]. Researchers have also introduced new basic displacement functions and used them to develop finite element schemes for non-prismatic Timoshenko beams [19-21]. In most studies of this sort, small deflections are assumed and equations of equilibrium or motion are developed based on the undeformed geometry.

In problems dealing with vibration, when the amplitude of a pulse load on a given beam exceeds a certain threshold, large amplitude vibrations are expected during which the deformed geometry deviates substantially from the undeformed state and the equations of motion must be derived in the deformed state. Several studies have been concerned with large-amplitude vibrations and their distinct features, such as the dependence of eigenfrequencies and natural modes on amplitude and chaos [22-24]. Nonlinear dynamic 
analyses were conducted on Timoshenko beam-columns on the Winkler foundation by Sapountzakis and Kampitsis [25].

Numerous advanced numerical methods are applied to study the nonlinear vibrations of a Timoshenko beam. For instance, Shahlaei-Far et al [26] studied the fourth-order nonlinear free vibrations of cantilever Timoshenko beams using the method of homotopy. They derived analytical expressions for the nonlinear eigenfrequencies and studied the effects of rotatory inertia, shear deformation, and slenderness ratio on the response. Zhong and Liao [27] used quartic B-splines in a spline-based differential quadrature method (SDQM) to study the higher-order nonlinear vibration of the Timoshenko beam. They concluded that some higherorder nonlinear frequencies decrease with increasing the ratio of the amplitude of vibration to the radius of gyration. In another study [28], the same authors investigated tapered Timoshenko beams and presented expressions for nonlinear fundamental frequencies of simply-supported and fully-clamped beams. Torabi et al [29] used a variational iteration method to study nonlinear vibrations of a Timoshenko beam and Ruta and Szybinski [30] applied different geometrical nonlinearity models to compare the nonlinear dynamics of a non-prismatic Timoshenko beam. Palacios [31] researched nonlinear normal modes of oscillation for anisotropic beams using both intrinsic equations where velocities and strains are primary degrees-of-freedom and Cosserat's exact description of the deformed geometry. On the basis of this description, nonlinear normal modes were obtained through the asymptotic approximation to the invariant manifolds.

Other specific studies were focused on one or two aspects of Timoshenko beam vibrations or on using a novel approach. For instance, Torabi et al. [29] considered an attached concentrated mass and studied the problem by the variational method; Radgolchin and Moeenfard [32] focused on a fully-clamped functionally-graded Timoshenko micro beam due to its application in MEMS; and Chakrabarti et al. [33] studied large amplitude free vibrations of a rotating nonhomogeneous beam with nonlinear spring and mass system. Simsek [34] studied nonlinear free vibration of a functionally-graded nanobeam on the base of the nonlocal strain gradient theory and a novel Hamiltonian approach as well as Hsieh et al. [35] developed and applied invariant manifold techniques to study large amplitude vibration of a cantilever beam. 
The study of nonlinear oscillations of structures with different dynamic states requires utilizing high-precision computational algorithms. Finite element methods realized in modern commercial packages (ANSYS, ABAQUS, etc.) form a strong suite for simulation of the static and dynamic response of structural and mechanical systems with high accuracy. Because analytical methods and experimental data dealing with the analysis of nonlinear structural models can rarely be applied, the only way to verify the computational results is to use different numerical approaches to solve the same problem. In this study, we develop a discrete model of the Timoshenko beam to study its large amplitude motion based on the finite difference method (FDM) as proposed in [36]. The scheme reduces governing partial differential equations to a system of ordinary differential equations of the first order. The approach is applied to derive the structural characteristics of the Timoshenko beam, and allows for parametric characterization. The objective of this study is to elucidate the discernable natural frequencies and vibration modes for linear and non-linear vibrations of the beam. Another aspect to be considered relates to the response and beam reaction to external pulse loads. The reduced model proposed minimizes computational costs, and at the same time, is not inferior in the accuracy of the results to the commercial FE software ABAQUS [37]. The paper, therefore, is an effort to introduce a computationally moderate model of reasonable accuracy to study large-amplitude vibration in deep beams.

The paper is structured as follows: following this terse introduction, Section 2 deals with the statement of the problem where the equations of dynamics of a geometrically nonlinear Timoshenko beam are derived using the extended Hamilton's principle (Lagranged'Alembert principle). The numerical finite-difference scheme for solving the equations of beam motion is presented in Section 3. Section 4 deals with the application of the developed scheme to the study of structural characteristics of the Timoshenko beam that simulates a non-prismatic beam. In Section 5, the vibrations of a non-prismatic beam simulating e.g. a simplified model of a composite turbine blade and the two-dimensional Timoshenko beam under action of different external pulse loads are calculated using both the MATLAB code developed on the basis of FDM and ABAQUS. The obtained time histories of the free end of the small beam are compared at different durations of the external load and the comparisons are discussed. Conclusions are given in Section 6. 


\section{Statement of the problem}

Let us consider an initially straight planar Timoshenko cantilever beam of length $L$, the left end of which is clamped and the right end is free, as depicted in Figure 1a. The beam is assumed to be made of a linearly elastic isotropic material and vibrates in one of the principal planes under the action of transverse load, continuously distributed on the plane of vibrations. Thus a geometrically nonlinear Timoshenko beam described in Weaver et al. [38] is considered. When deriving the beam's mathematical model, we will take into account the shear and bending deformations as well as the lateral and rotatory inertia of the beam, but at the same time, ignore the axial force and deformations which are normally neglected in a beam theory.

A Cartesian coordinate system is defined such that axis $O x$ is the intersection of two mutually orthogonal planes of inertia of the beam as illustrated in Figure 1a. Note that the axis coincides with the beam's neutral axis. The transversal vibrations of the beam are considered in the coordinate plane $O x y$, which is combined with one of its inertial planes. The origin of the coordinate system coalesces with the clamped end of the beam. The transverse displacement field of the beam is denoted by $w(x, t)$, where $x$ is the spatial coordinate along the beam in the undeformed configuration ranging from 0 to length $L$ and $t$ is the time.

(a)

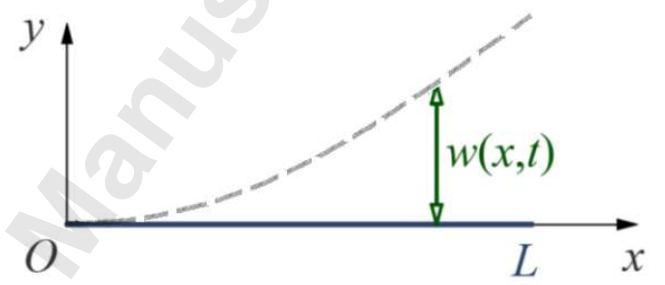

(b)

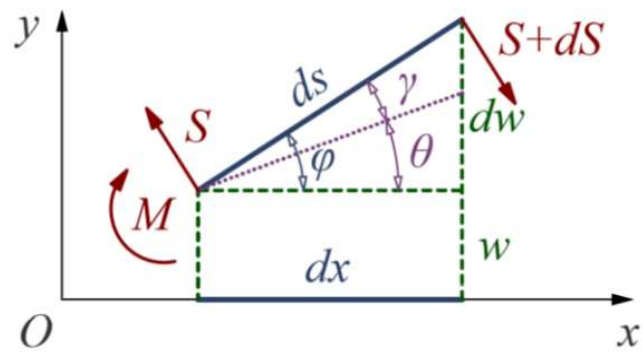

Figure 1. (a) Configuration of the beam in the coordinate system $O x y$ : the horizontal solid line depicts the initial configuration of the beam, where the dashed curve shows the deformed shape of the beam at time $t$. (b) Undeformed $(d x)$ and deformed $(d s)$ beam elements are illustrated, where $\varphi$ is the slope angle, $\theta$ is the rotation angle, and $\gamma$ is the shear angle of deformation. Internal forces and moments are represented by shear force $S$ and bending moment $M$. 
The transverse displacement field of the beam is denoted by $w(x, t)$, where $x$ is the spatial coordinate along the beam in the undeformed configuration ranging from 0 to length $L$ and $t$ is the time. Since the concept of geometric curvature is applied in nonlinear bending deformation analysis, the curvature of the beam $(\kappa)$ is considered which, by referring to Figure $1 \mathrm{~b}$, is defined by the following equation:

$$
\kappa=\frac{d \theta}{d s}=\frac{d \theta}{d x} \frac{d x}{d s}=\theta^{\prime} \frac{d x}{\sqrt{d x^{2}+d w^{2}}}=\frac{\theta^{\prime}}{\sqrt{1+w^{\prime 2}}},
$$

where $\theta$ is the rotation of the beam cross-section, $d x$ and $d s$ are the length of the undeformed and deformed beam elements, respectively, and the prime denotes derivative with respect to $\left.x()^{\prime}=\partial() / \partial x\right)$. The position of a beam element during oscillation is determined by the displacement of its neutral axis passing through its centroid, and the slope angle $\varphi$ of the beam axis in the plane $O x y$. For a Timoshenko beam, the angle $\varphi$ depends not only on rotation of the beam element under the action of bending moments but also on shear deformation angle $\gamma$ (see Figure 1b):

$$
\varphi=\tan ^{-1} w^{\prime}=\gamma+\theta .
$$

Since axial displacements of the beam are neglected, the mathematical model describing the beam motion will include only the effects of the shear force $S$ and the bending moment $M$ designated in Figure 1b. The development of this model is based on the variational method assuming the following linear constitutive equations of the theory of elasticity hold, as only geometric nonlinearity is considered here.

$$
S=\alpha G A \gamma \quad \text { and } \quad M=E I \kappa,
$$

where $\alpha$ is the shear coefficient which depends only on the shape of the beam cross-section; $E$ and $G$ are the elastic and shear moduli, respectively; $A(x)$ is the cross-sectional area; and $I(x)$ is the second moment of inertia of the beam cross-section. As a result, $E I$ and $G A$ are the shear and bending stiffness, respectively.

Taking into account equation (3), one can write the potential energy of bending and shear deformations of the Timoshenko beam in the following additive form: 


$$
U=U_{b}+U_{s}=\frac{1}{2} \int_{0}^{L} E I \kappa^{2} d x+\frac{1}{2} \int_{0}^{L} G A \gamma^{2} d x
$$

Similarly, the kinetic energy of transverse and rotational movements of the beam add up to the total kinetic energy as

$$
T=T_{t}+T_{r}=\frac{1}{2} \int_{0}^{L} \rho A \dot{w}^{2} d x+\frac{1}{2} \int_{0}^{L} \rho I \dot{\theta}^{2} d
$$

where $\rho$ is the density and the overdot denotes the time derivative.

In our approach, the external force acting on the beam is represented as follows:

$$
q(x, t)=p(x, t) C(x) \cos w^{\prime},
$$

where $c(x)$ is the beam breadth and may in general be a function of variable $x$ and $p(x, t)$ is the external pressure applied on the beam. Then the virtual work of the external force will be:

$$
\delta w_{n c}=\int_{0}^{L} p(x, t) C(x) \cos w^{\prime} \delta w d x
$$

The extended principle of least action is applied for valid trajectories as:

$$
\delta S=\int_{t_{1}}^{t_{2}}\left(\delta T-\delta U+\delta w_{n c}\right) d t=0,
$$

where $\delta T$ and $\delta U$ are the appropriate variations of kinetic (5) and potential (4) energies. From (8), performing standard transformations and taking into account the independence of variations of $\delta w$ and $\delta \theta$, one obtains the following governing partial differential equations of beam vibration:

$$
\begin{gathered}
-\rho A \ddot{w}+\frac{(E I)^{\prime} \theta^{\prime 2} w^{\prime}+2 E I \theta^{\prime \prime} \theta^{\prime} w^{\prime}+E I \theta^{\prime 2} w^{\prime \prime}}{\left(1+w^{\prime 2}\right)^{2}}-\frac{4 E I \theta^{\prime 2} w^{\prime 2} w^{\prime \prime}}{\left(1+w^{\prime 2}\right)^{3}} \\
+\frac{(\alpha G A)^{\prime}\left(\tan ^{-1} w^{\prime}-\theta\right)+(\alpha G A)\left(\frac{w^{\prime \prime}}{1+w^{\prime 2}}-\theta^{\prime}\right)}{\left(1+w^{\prime 2}\right)} \\
-\frac{2 w^{\prime} w^{\prime \prime}\left(\alpha G A\left(\tan ^{-1} w^{\prime}-\theta\right)\right)}{\left(1+w^{\prime 2}\right)^{2}}+p(x, t) C(x) \cos w^{\prime}=0,
\end{gathered}
$$

and

$$
-\rho I \ddot{\theta}+\frac{\left(E I \theta^{\prime}\right)^{\prime}\left(1+w^{\prime 2}\right)-2 w^{\prime} w^{\prime \prime}\left(E I \theta^{\prime}\right)}{\left(1+w^{\prime 2}\right)^{2}}+\alpha G A\left(\tan ^{-1} w^{\prime}-\theta\right)=0
$$


with natural boundary conditions at cantilever beam ends that include the shear force:

$$
\left[\left(\frac{E I \theta^{\prime 2} w^{\prime}}{\left(1+w^{\prime 2}\right)^{2}}-\frac{\alpha G A\left(\tan ^{-1} w^{\prime}-\theta\right)}{\left(1+w^{\prime 2}\right)}\right) \delta w\right]_{0}^{L}=0
$$

and the bending moment:

$$
\left[\frac{-E I \theta^{\prime}}{\left(1+w^{\prime 2}\right)} \delta \theta\right]_{0}^{L}=0
$$

Relations (11) and (12) provide the boundary conditions for the problem under consideration:

$$
\begin{gathered}
w=0 \text { and } \theta=0 \quad \text { at } x=0, \\
\frac{E I \theta^{\prime 2} w^{\prime}}{\left(1+w^{\prime 2}\right)^{2}}-\frac{\alpha G A\left(\tan ^{-1} w^{\prime}-\theta\right)}{1+w^{\prime 2}}=0, \quad-\frac{E I \theta^{\prime}}{1+w^{\prime 2}}=0 \quad \text { at } x=L .
\end{gathered}
$$

The initial-boundary value problem is supplemented by the initial conditions:

$$
w(x, 0)=\dot{w}(x, 0)=\theta(x, 0)=\dot{\theta}(x, 0)=0 .
$$

The presented mathematical model should be supplemented with input data including the beam geometry and mechanical characteristics of the material which the beam is made of. We consider a homogeneous elastic beam with the following mechanical properties: $\rho=$ $7800 \mathrm{~kg} / \mathrm{m}^{3}, E=2 \times 10^{11} \mathrm{~Pa}$, and $v=0.3$, where $v$ is the Poisson's ratio of the beam material. The cross-section area along the beam $A(x)$ and the second moment of inertia $I(x)$ are set on the basis that the beam simulates the non-prismatic Timoshenko beam.

\section{Numerical modelling}

Due to the complexity of the partial differential equations governing the non-linear dynamics of the Timoshenko beam, in general, analytical solutions for the PDE's do not exist and they will be solved numerically. To derive the dimensionless form of Eqs. (9) and (10), we introduce the following relations:

$$
\begin{gathered}
\bar{w}=\frac{w}{h}, \quad \bar{x}=\frac{x}{L}, \quad \bar{t}=\frac{t}{\tau}, \quad \text { where } \quad \tau=\frac{L}{c}, \quad c=\sqrt{\frac{E}{\rho}}, \\
\bar{A}=\frac{A}{h^{2}}, \quad \bar{I}=\frac{I}{h^{4}}, \quad \bar{C}=\frac{C}{h}, \quad \text { and } \bar{p}=\frac{L^{2}}{h^{2} E} p .
\end{gathered}
$$


Here $h$ is the conditional width of the beam. It is assumed that $h=\sqrt{\overline{A(x)}}$, where the bar denotes the mean value (further bars over non-dimensional parameters will be omitted). Introducing the notations:

$$
\lambda=\frac{L}{h} \quad \text { and } \quad D=\frac{G}{E}=\frac{1}{2(1+v)},
$$

and damping factor $\varepsilon_{1}=\varepsilon L / c$ for variable $w$ we obtain the dimensionless equations of the beam motion, which may be written in the following operator form:

$$
\begin{gathered}
\ddot{w}+\varepsilon_{1} \dot{w}=L_{1}(w, \theta)+p(x, t) \frac{C(x)}{A(x)} \cos \left(\frac{1}{\lambda} w^{\prime}\right), \\
\ddot{\theta}=L_{2}(w, \theta) .
\end{gathered}
$$

Here $L_{1}$ and $L_{2}$ are the operators of elastic and mass forces acting to the beam defined as follows:

$$
\begin{aligned}
& L_{1}(w, \theta)=\frac{1}{\lambda^{2}} \frac{I}{A} \frac{\left(I^{\prime} / I\right) \theta^{\prime 2} w^{\prime}+2 \theta^{\prime} \theta^{\prime \prime} w^{\prime}+\theta^{\prime 2} w^{\prime \prime}}{\left(1+\left(\frac{1}{\lambda} w^{\prime}\right)^{2}\right)^{2}}-\frac{1}{\lambda^{4}} \frac{I}{A} \frac{4 \theta^{\prime 2} w^{\prime 2} w^{\prime \prime}}{\left(1+\left(\frac{1}{\lambda} w^{\prime}\right)^{2}\right)^{3}} \\
& +\alpha D \lambda \frac{\left(A^{\prime} / A\right)\left(\tan ^{-1}\left(\frac{1}{\lambda} w^{\prime}\right)-\theta\right)+\left(\frac{\frac{1}{\lambda} w^{\prime \prime}}{1+\left(\frac{1}{\lambda} w^{\prime}\right)^{2}}-\theta^{\prime}\right)}{1+\left(\frac{1}{\lambda} w^{\prime}\right)^{2}} \\
& -\alpha D \frac{1}{\lambda} \frac{2 w^{\prime} w^{\prime \prime}\left(\tan ^{-1}\left(\frac{1}{\lambda} w^{\prime}\right)-\theta\right)}{\left(1+\left(\frac{1}{\lambda} w^{\prime}\right)^{2}\right)^{2}}
\end{aligned}
$$

and

$$
L_{2}(w, \theta)=\frac{\left(\left(I^{\prime} / I\right) \theta^{\prime}+\theta^{\prime \prime}\right)\left(1+\left(\frac{1}{\lambda} w^{\prime}\right)^{2}\right)-\frac{2}{\lambda^{2}} w^{\prime} w^{\prime \prime} \theta^{\prime}}{\left(1+\left(\frac{1}{\lambda} w^{\prime}\right)^{2}\right)^{2}}+\alpha D \lambda^{2} \frac{A}{I}\left(\tan ^{-1}\left(\frac{1}{\lambda} w^{\prime}\right)-\theta\right)
$$

Also if one takes into account the facts that $E I \neq 0$ and $1+w^{2} \neq 0$ in condition (14), the boundary conditions at the free beam end are obtained in the following form:

$$
\theta^{\prime}=0 \quad \text { and } \quad \tan ^{-1} \frac{w^{\prime}}{\lambda}-\theta=0 \quad \text { at } x=1
$$


In the numerical scheme, PDEs (18) and (19) are reduced to ODEs with variable time by applying the finite difference method. So, we transfer from the distributed-parameter system (continuous model) to a finite-dimensional lumped-parameter system (discrete system) which greatly simplifies the problem of determining the dynamic characteristics of the beam.

We introduce the uniform grid $G_{N}=\left\{0 \leq x_{i} \leq 1, \quad x_{i}=1 / N, i=0, \ldots, N\right\}$ along the beam and use an approximation $O\left(\Delta x^{2}\right)$ for spatial derivatives, where $\Delta x$ is the computational step regarding the spatial coordinate. As a result, the following system of ODEs in the internal nodes is derived:

$$
\begin{gathered}
\ddot{w}_{i}+\varepsilon_{1} \dot{w}_{i}=L_{1, \Delta x}\left(w_{i-1}(t), \theta_{i-1}(t), w_{i}(t), \theta_{i}(t), w_{i+1}(t), \theta_{i+1}(t)\right) \\
+p\left(x_{i}, t\right) \frac{C\left(x_{i}\right)}{A\left(x_{i}\right)} \cos \left(\frac{1}{\lambda} w_{i}{ }^{\prime}(t)\right), \\
\ddot{\theta}_{i}=L_{2, \Delta x}\left(w_{i-1}(t), \theta_{i-1}(t), w_{i}(t), \theta_{i}(t), w_{i+1}(t), \theta_{i+1}(t)\right), \quad i=1, \ldots, N-1 .
\end{gathered}
$$

Eqs. (23) and (24) are transformed to $4(N-1)$ differential equations of the first order regarding variables $w, \theta, \dot{w}, \dot{\theta}$ with the following boundary conditions:

$$
\begin{gathered}
w_{0}=0, \quad \theta_{0}=0, \quad \dot{w}_{0}=0, \quad \text { and } \quad \dot{\theta}_{0}=0 . \\
w_{N}=\frac{2}{3} \lambda \Delta x \tan \theta_{N}+\frac{1}{3}\left(4 w_{N-1}-w_{N-2}\right) \quad \text { and } \quad \theta_{N}=\frac{1}{3}\left(4 \theta_{N-1}-\theta_{N-2}\right) .
\end{gathered}
$$

Note that expression (26) follows the approximation of spatial derivative at the last grid node by the right-hand discrete operator of the second order.

\section{Eigenfrequency analysis}

Natural vibrations of the beam are described by equations (23) and (24) when $p\left(x_{i}, t\right)=0$ and $\varepsilon=0$ and boundary conditions are as equations (25) and (26). These equations are a finite-difference approximation of the mixed initial-boundary-value problem defined by equations (9), (10), (13), and (14), which depends on the spatial resolution of the grid $G_{N}$. We settle convergence of the numerical scheme when calculating eigenfrequencies of the linear problem for the present beam. It is described by the following equations: 


$$
\begin{gathered}
\ddot{w}=\sum_{k=-1}^{1}\left(\frac{\partial L_{1, \Delta x}}{\partial w_{i+k}}\right)_{0} w_{i+k}+\sum_{k=-1}^{1}\left(\frac{\partial L_{1, \Delta x}}{\partial \theta_{i+k}}\right)_{0} \theta_{i+k} \\
\ddot{\theta}=\sum_{k=-1}^{1}\left(\frac{\partial L_{2, \Delta x}}{\partial w_{i+k}}\right)_{0} w_{i+k}+\sum_{k=-1}^{1}\left(\frac{\partial L_{2, \Delta x}}{\partial \theta_{i+k}}\right)_{0} \theta_{i+k}, \quad i=1, \ldots, N-1 .
\end{gathered}
$$

Index " 0 " in Eqs. (27) and (28) means that the Jacobian of the system (23)-(24) is calculated at $w_{i}=0, \theta_{i}=0$. Note also that values of the functions $A(x)$ and $I(x)$ in the grid nodes $x_{i}$ are estimated by the piecewise cubic Hermite polynomial interpolation. The problem is considered for a rectangular section with $v=0.3$ and $\alpha=5 / 6$.

Dependencies of the first natural frequencies of the beam on a number of grid nodes $N$ are shown in Figure 2. One can see that the process of calculating these values at $N=300$ and $N=600$ is convergent with the relative errors of $2.86 \%, 5.99 \%$, and $7.98 \%$ for the first $\omega_{1}$, second $\omega_{2}$, and third $\omega_{3}$ eigenfrequencies, respectively. In subsequent calculations, the grid $G_{300}$ will be used, which covers all the features of the input functions $A(x)$ and $I(x)$, and is a good choice between the accuracy of computing and the economy of computational resources (See Fig. 2). Note that the stiffness matrix of the linear problem at $N=300$ is positive definite. This means that the corresponding linear oscillations of the beam are stable.

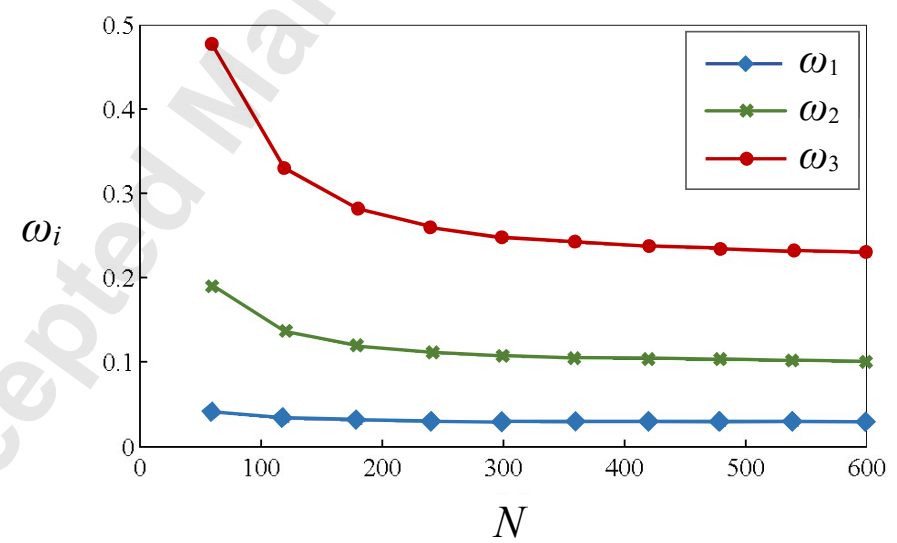

Figure 2. Evaluation of convergence of the numerical scheme for the natural frequencies of the beam

Three computed natural frequencies of the Timoshenko beam with the specified mechanical and geometrical properties are presented in Table 1 in both dimensionless and dimensional forms. These correlate perfectly with the results obtained from the commercial software ABAQUS. The mode shapes of the displacement $w$ and rotation angle $\theta$ corresponding to 
the natural frequencies $\omega_{1}$ and $\omega_{2}$ are depicted in Figure $3 a$ and $b$, respectively, which correspond with the ones obtained from ABAQUS as shown in Figure 3c.

Table1. Computed natural frequencies for the Timoshenko beam

\begin{tabular}{|c|c|c|c|}
\hline Mode number & 1 & 2 & 3 \\
\hline Frequency & $0.029(1.3 \mathrm{~Hz})$ & $0.106(4.7 \mathrm{~Hz})$ & $0.250(11 \mathrm{~Hz})$ \\
\hline
\end{tabular}
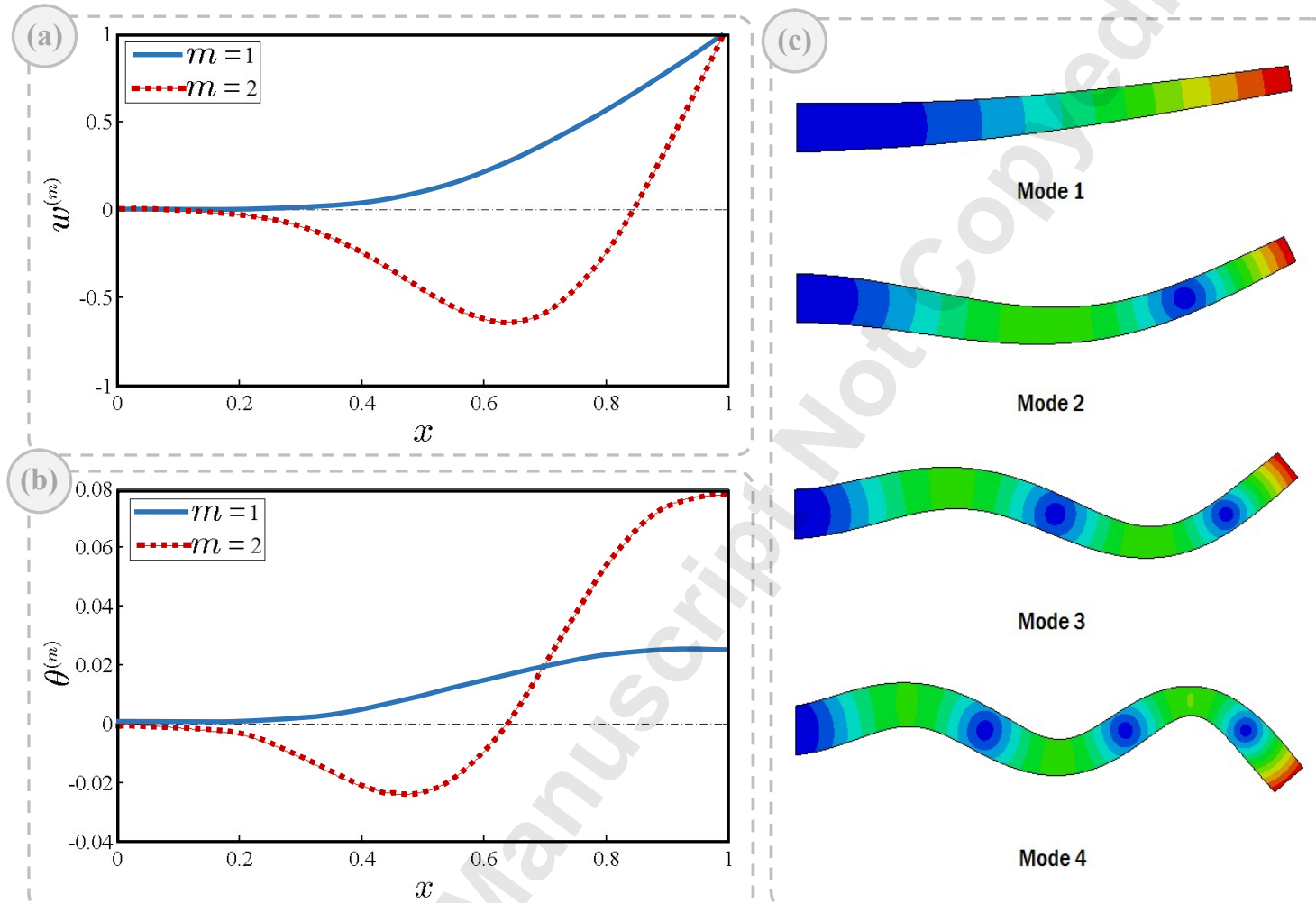

(d)

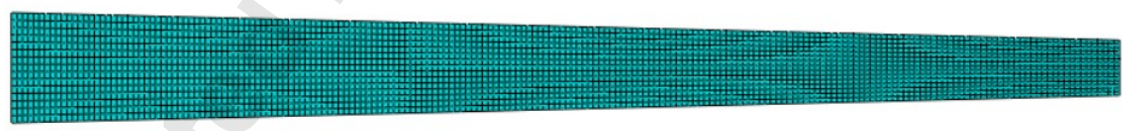

Mode 4

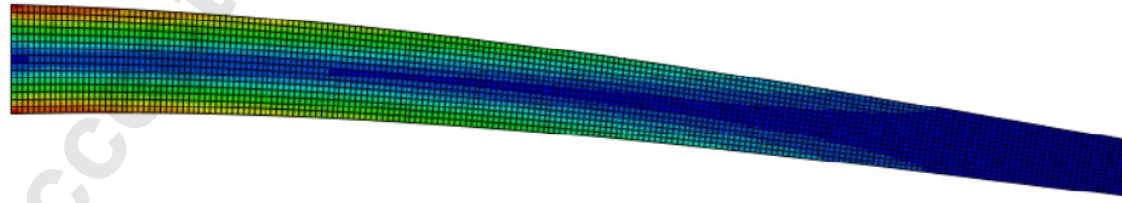

Figure 3. Computed modal shapes of (a) the transversal displacement and (b) the angle of axial rotation for the first and second natural frequencies of the beam obtained by FDM. (c) Natural modes of vibration for the first four modes of the beam from ABAQUS. (d) Finite element mesh of the model (converged mesh size is $0.005 \mathrm{~m}$ ). 
In order to study the nonlinear eigen behaviour of the Timoshenko beam, we introduce the initial nonzero beam deflections $w(x, 0), \theta(x, 0)$ with zero velocities $\dot{w}(x, 0), \dot{\theta}(x, 0)$. The spectrum of the free vibrations that the beam undergoes is used to identify the frequency of its non-linear motion. As the initial deviations, the obtained modes of linear oscillations are considered:

$$
w_{i}(0)=R \cdot w_{i}^{(m)} \text { and } \theta_{i}(0)=R \cdot \theta_{i}^{(m)} \quad(i=1, \ldots, N-1),
$$

where $w_{i}^{(m)}$ and $\theta_{i}^{(m)}$ are the $m^{\text {th }}$ mode of linear vibrations that corresponds to the natural frequency $\omega_{m}$. Note that the linear modes are normalized so that the displacement at node $N-1$ is unity i.e. $w_{N-1}^{(m)}=1$. It follows that $R$ is the amplitude of vibrations at the point of $x_{N-1}$. Equations (15) are supplemented by the following conditions for derivatives:

$$
\dot{w}_{i}(0)=0 \text { and } \quad \dot{\theta}_{i}(0)=0 \quad(i=1, \ldots, N-1) .
$$

To calculate the eigenfrequency of non-linear vibrations of the beam, we will analyze time series of the transverse displacement $w$ in the beam point of $x_{N-1}$, since the amplitude of $w$ is maximal there. Fig. 4 illustrates the dependencies of $w_{N-1}(t)$ for the first (Fig. 4a) and second (Fig. 4b) modes. One can see that the periodic process is stable for both the first and second modes. It is necessary to mention that similar dependencies, but with smaller amplitudes, occur in the case of other points of the beam and for higher modes.

(a)

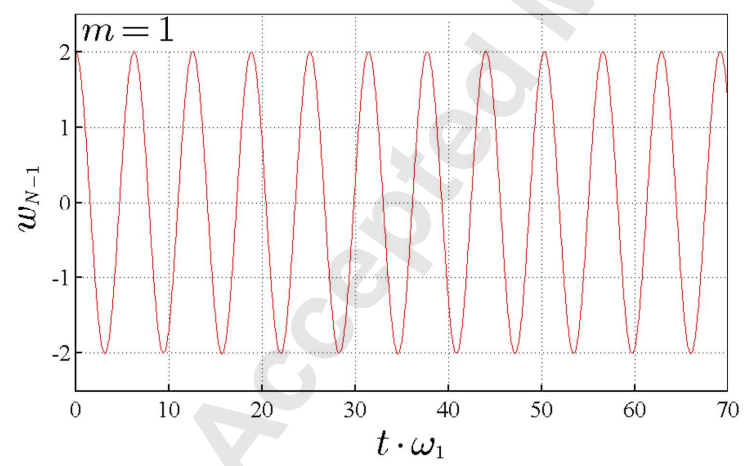

(b)

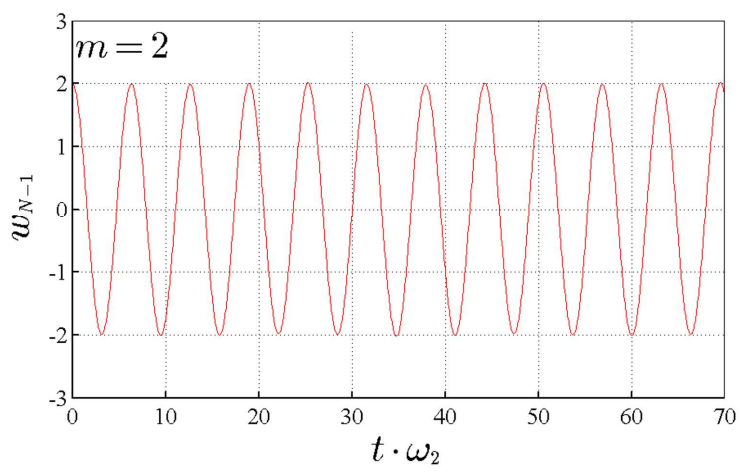

Figure 4. Dependencies of $w_{N-1}(t)$ with non-linear beam vibrations for (a) the first mode, and (b) the second mode.

It is a well-known fact that nonlinear frequencies of a system are amplitude-dependent, i.e. there is a frequency of beam vibrations for each amplitude $R$ and by changing $R$ in a wide 
range one derives the skeleton curve that demonstrates the correlation between the eigenfrequency and the amplitude of non-linear vibrations of the beam for a given mode. Fig. 5 illustrates the skeleton curves for variable $w$ corresponding to the first and second modes of nonlinear vibrations of the Timoshenko beam. It is seen that both curves are smooth in nature and decreasing i.e. the eigenfrequency decreases with the rising amplitude of nonlinear oscillations.

(a)

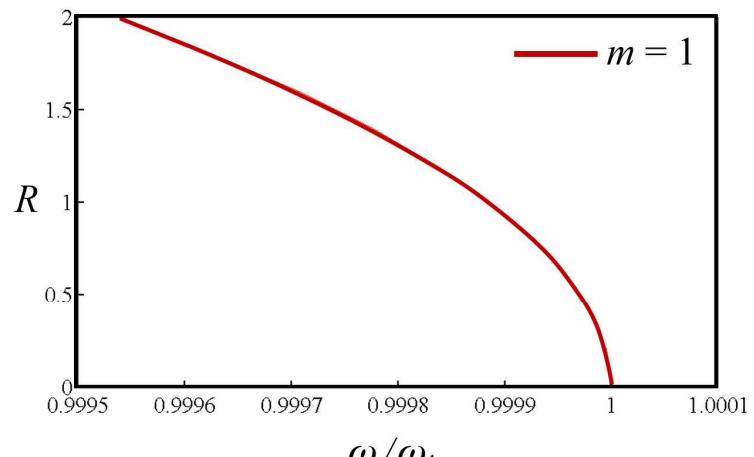

(b)

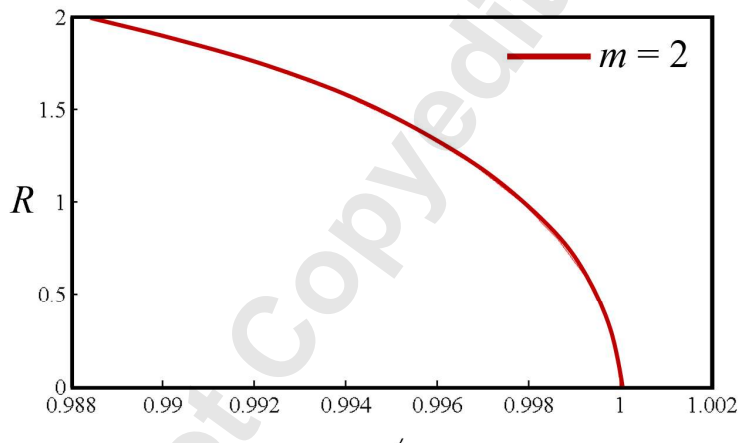

$\omega / \omega_{2}$

Figure 5. Skeleton curves matching (a) the first mode and (b) the second mode of the Timoshenko beam

\section{Forced beam vibrations}

The algorithm developed was applied to the simulation of forced vibrations of the beam in order to clarify its reaction to the periodic transversal load oscillating with the frequency that is close to the fundamental beam frequency $\omega_{1}$. The external force $p(x, t)$ is assumed to take a harmonic temporal form and be distributed uniformly along the beam axis in accordance with the last term of Eq. (9), thus:

$$
p(x, t)=q_{0} \sin \theta \omega_{p} t .
$$

where $q_{0}$ and $\omega_{p}$ are the force amplitude and excitation frequency, respectively.

Equations (23) and (24) with boundary conditions (25) and (26) along with zero initial conditions (15) are considered for the following fixed parameters: $q_{0}=0.0015, \omega_{p} / \omega_{1}=1.05$, and $\varepsilon=0.02$. Fig. 6 illustrates the time history of the transverse displacement $w$ in the beam axis points at dimensionless coordinates $x=1$ (Fig. 6a), $x=0.75$ (Fig. 6b), $x=0.5$ (Fig. 6c) and $x=0.25$ (Fig. 6d). It is seen that the beam points oscillate in different manners depending on their position. The points located near the free end are subject to almost harmonic oscillations and the free end vibrates at a single frequency close to the external 
force frequency. When a point approaches the pinched end of the beam, the nature of its forced oscillations becomes much more complicated. We deal with two-frequency oscillations here and oscillations of the points located closer to the pinched end are lagging in phase compared to oscillations of the free end due to viscous damping.
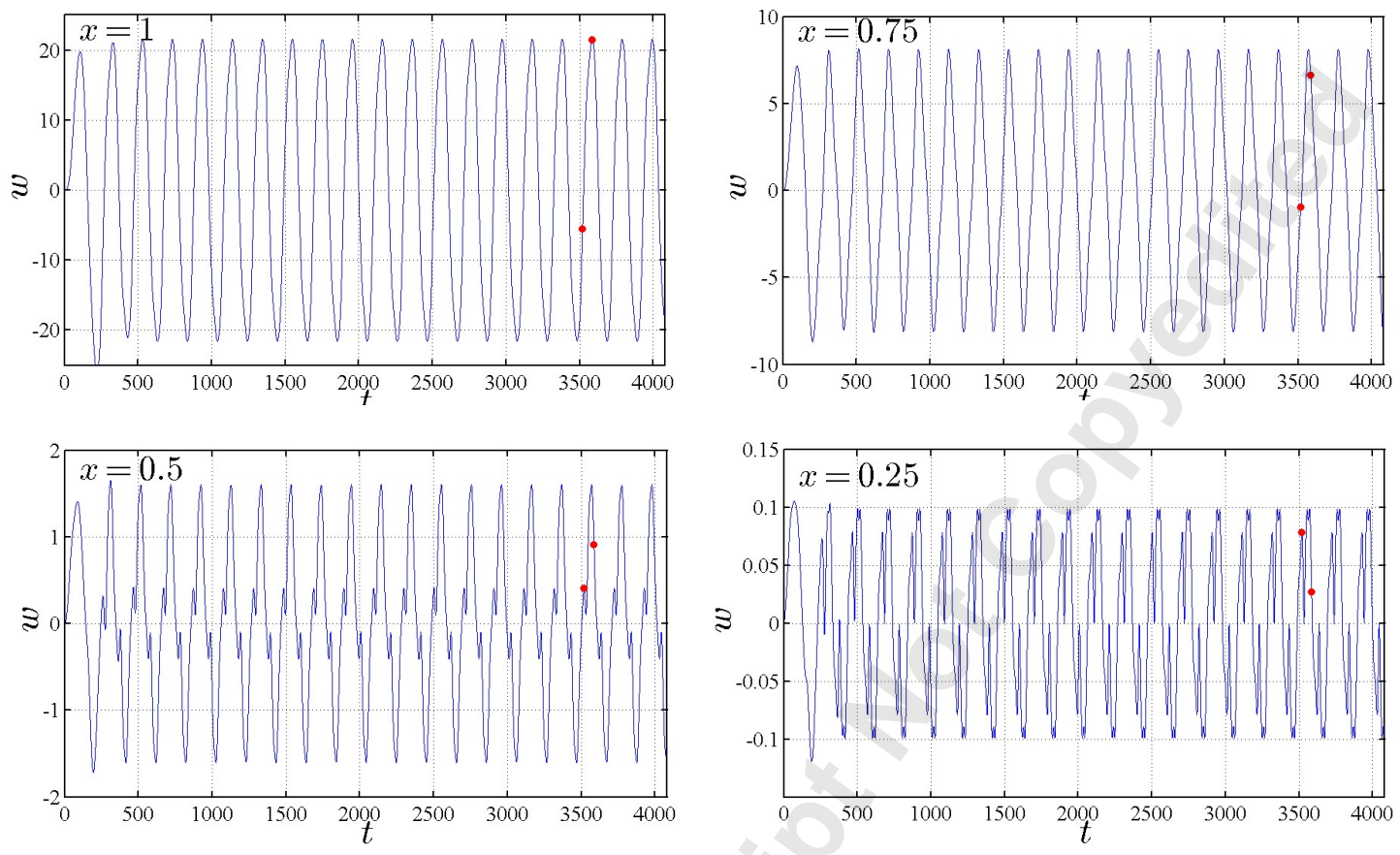

Figure 6. Time histories of the transverse displacement of the beam points under the action of the same periodic loading

Viscous damping and specific distribution of the external force along the beam axis lead to a change of the mode at which the beam oscillates with the effect of the former demonstrated in Fig.7. This figure depicts the beam shapes at two time instances. The first corresponds to the maximum amplitude of oscillations of the free end (green line) and the second lies near the middle of the period (blue line). These instances are shown with red markers on the graphs of function $w(x, t)$ in Fig. 6. It follows from Fig. 7 that near the first frequency $\omega_{1}$, the beam oscillates similarly to the second natural beam mode.

Further vibrations of the small two-dimensional cantilever beam under the action of different external pulse loads are calculated using both the MATLAB code developed on the basis of FDM and the finite element software package ABAQUS. The geometric and physical parameters of the beam are as follows: length $L=1 \mathrm{~m}$, thickness varies linearly from $0.1 \mathrm{~m}$ at $x=0$ to $0.05 \mathrm{~m}$ at $x=L, \rho=7800 \mathrm{~kg} / \mathrm{m}^{3}, E=2 \times 10^{5} \mathrm{MPa}, v=0.25, \alpha=5 / 6$, and $\varepsilon=0$. 
The uniform grid $G_{300}$ is used in the calculations by FDM. Initially, a linear analysis of this beam was performed using both FDM and ABAQUS. It was obtained that the natural period of the fundamental mode of the beam is equal to $11.3067 \mathrm{msec}$ as obtained by FDM and $11.2994 \mathrm{msec}$ as calculated by ABAQUS. One can see that the natural periods of the beam's fundamental mode derived by the two methods are almost the same. In subsequent simulations, the duration of the rectangular pulse load will be related to this period to cover the entire spectrum of the regime of loading. The impulsive regime with the pulse load duration of $2.2 \mathrm{msec}$, the dynamic regime with the duration equal to $11.3 \mathrm{msec}$, and the quasistatic one corresponding to a duration of $34 \mathrm{msec}$ will be considered.

Fig. 8 shows the resistance curves for the beam obtained through linear and nonlinear analyses conducted using ABAQUS. The curves depict the point at which geometric nonlinear phenomenon emerges and as deformations grow larger this effect becomes more significant. In Figs. 9 and 10, the time histories of the beam's endpoint are denoted for different amplitudes of the external load which is equal to $8 \mathrm{~N} / \mathrm{mm}$ in Fig. 9 and $12 \mathrm{~N} / \mathrm{mm}$ in Fig. 10. These are amplitudes which are large enough to allow for nonlinear effects to be pronounced. Fig. 9 demonstrates a good correlation between results obtained by the MATLAB code and ABAQUS especially at the initial stage of the calculations. The difference is further due to the fact that the calculations with FDM do not take into account the internal diffusion of the beam, which is especially evident at the higher load (Fig. 10). One can see in Figs. 9 and 10 that for moderate displacements a better correlation is obtained, however, even for large displacements a reasonable correlation exists. Figs. 11a and 11b demonstrate the dependence of the maximum displacement on the pulse duration and the maximum pressure, respectively. As can be seen from these figures, the nonlinear effects emerge at higher displacement levels. 


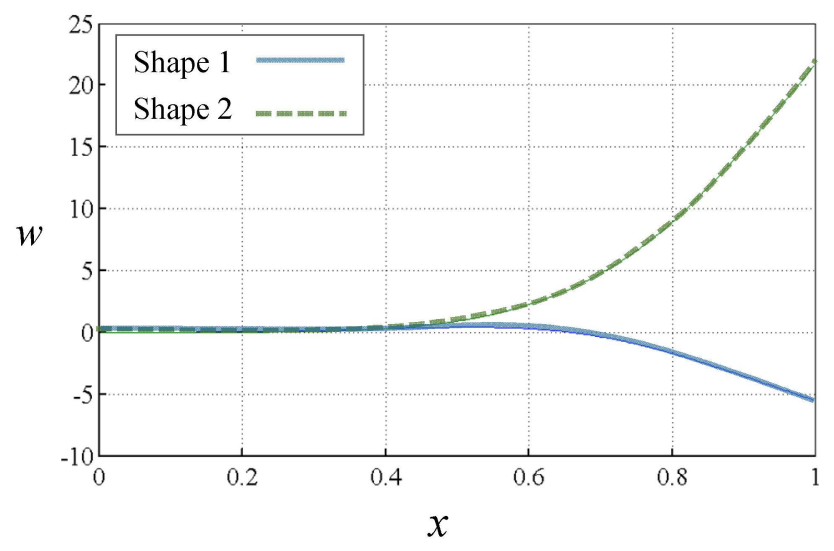

Figure 7. The shapes of the transversal displacement during the period of forced oscillations

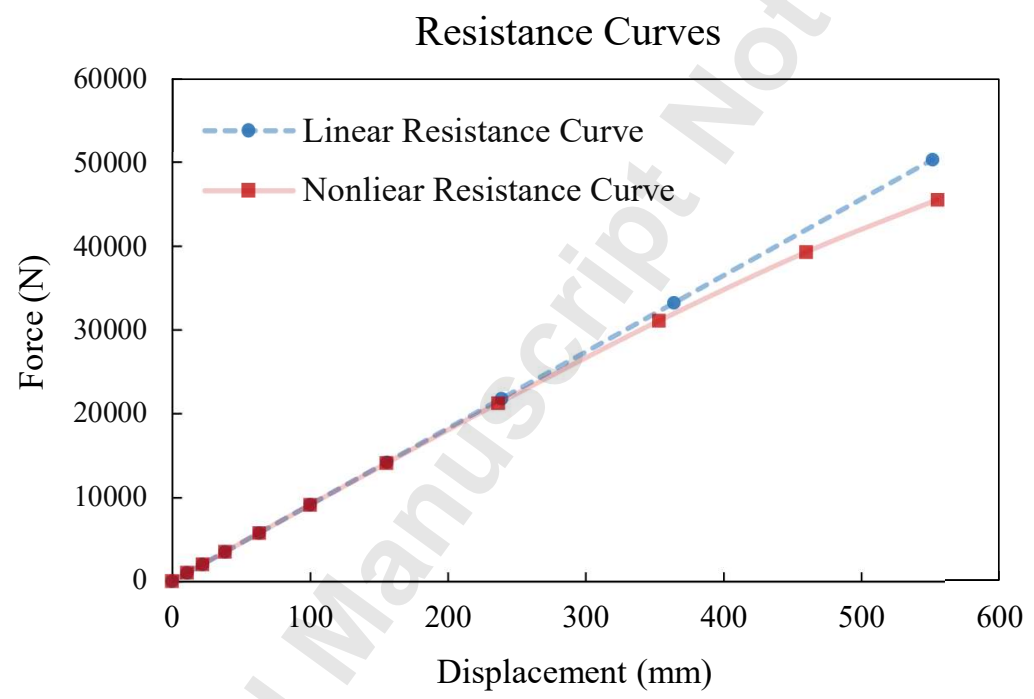

Figure 8. Reaction force versus tip displacement curve, showing the emergence of nonlinear behaviour in the beam 
(a)

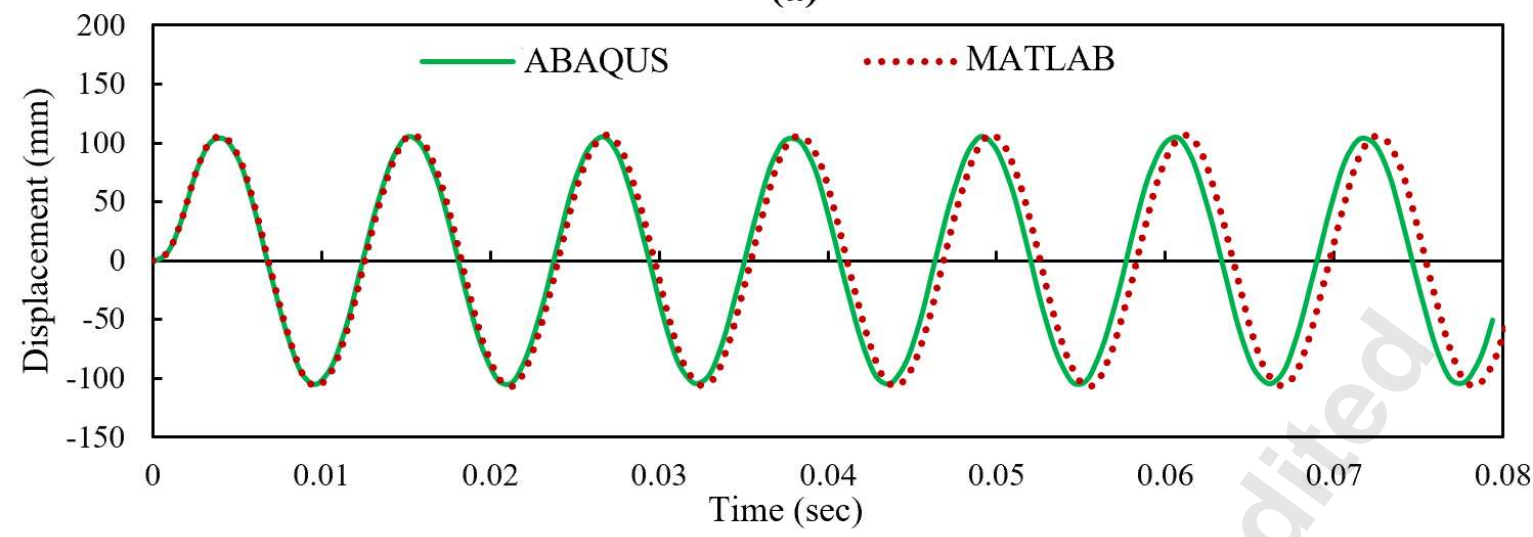

(b)

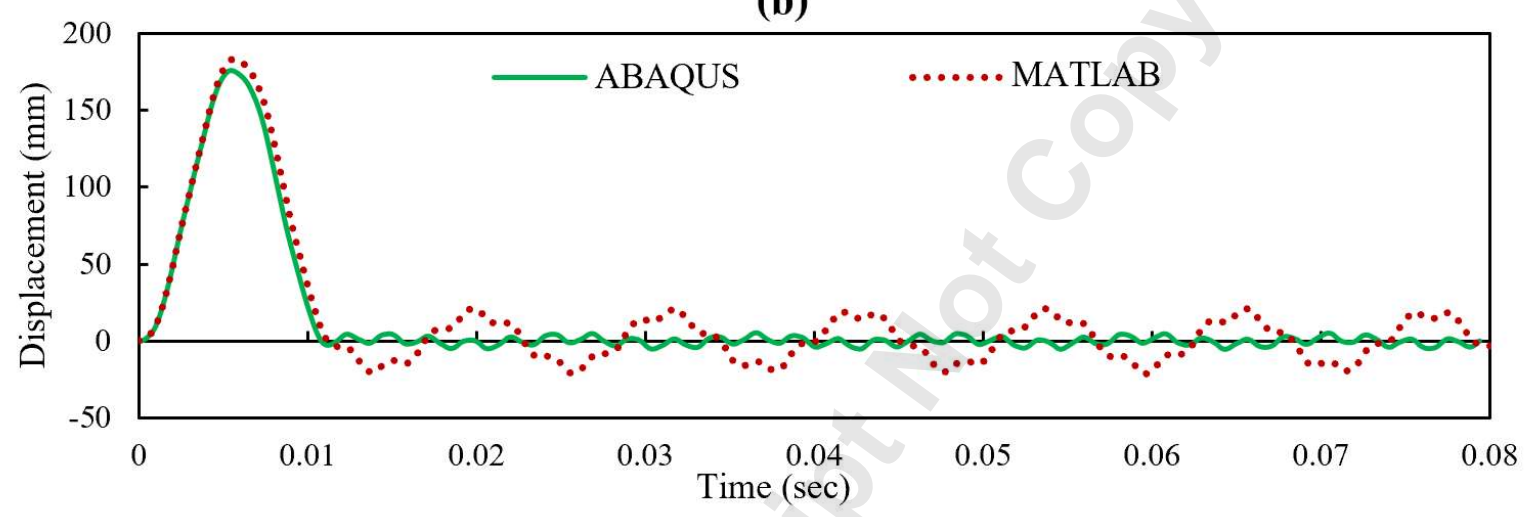

(c)

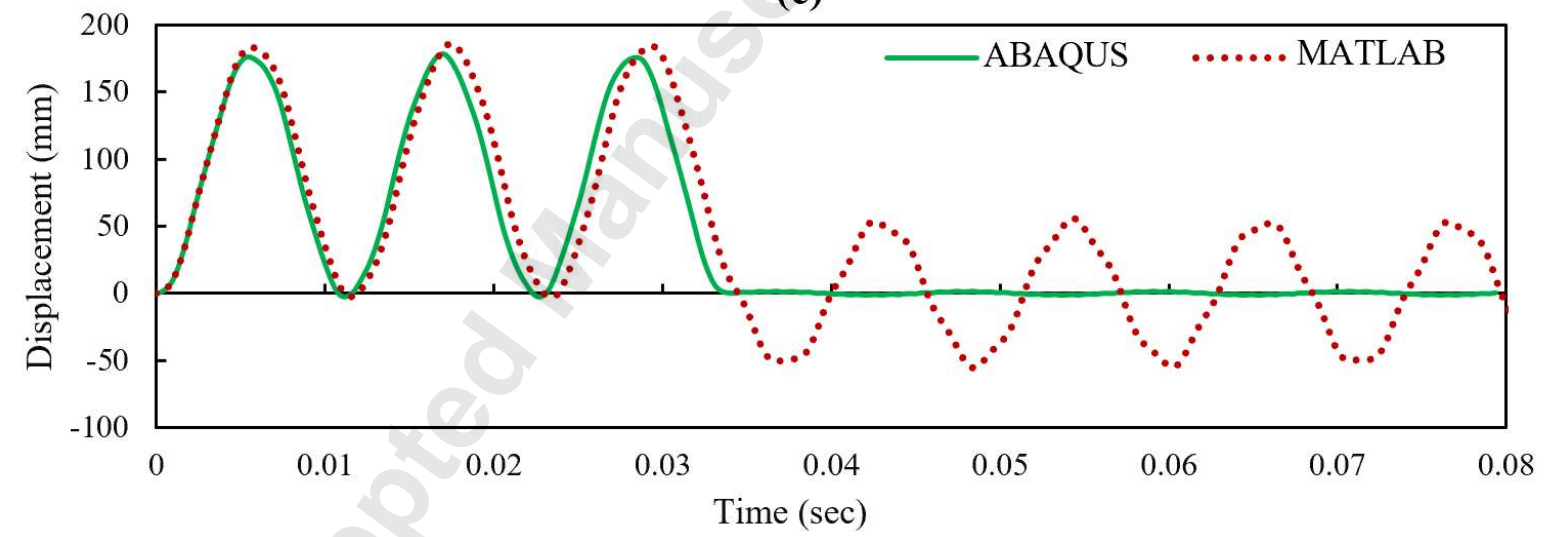

Figure 9. Correlation of displacement time histories between ABAQUS and the MATLAB code for (a) small, (b) medium, and (c) large deflections due to rectangular pulse load of magnitude $8 \mathrm{~N} / \mathrm{mm}$ and durations $2.2 \mathrm{msec}$ (impulsive), $10.1 \mathrm{msec}$ (dynamic) and $34 \mathrm{msec}$ (quasi-static). The full range of loading regimes is considered. 
(a)

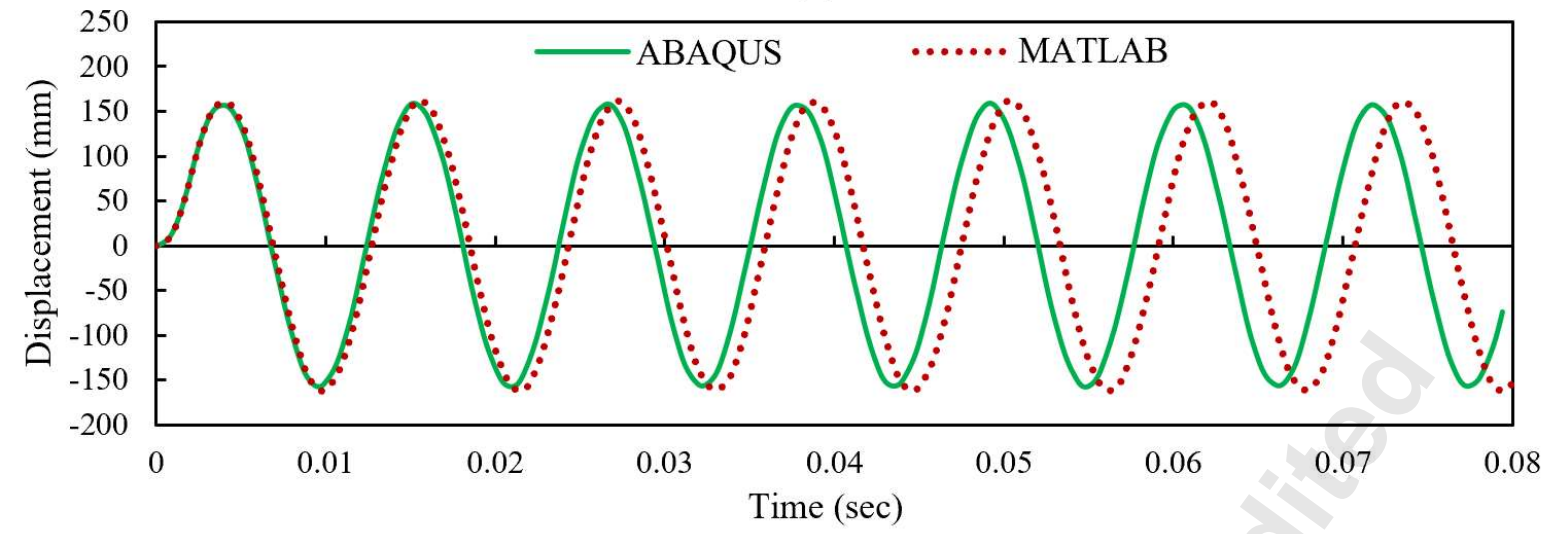

(b)

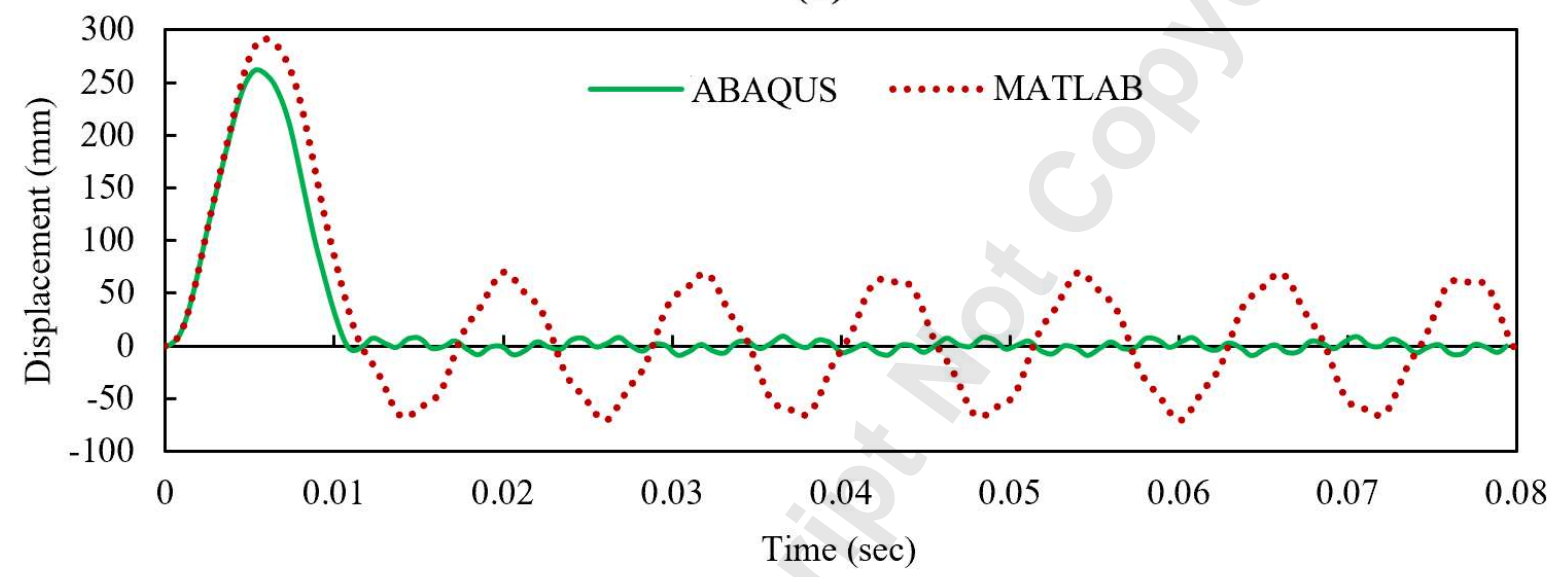

(c)

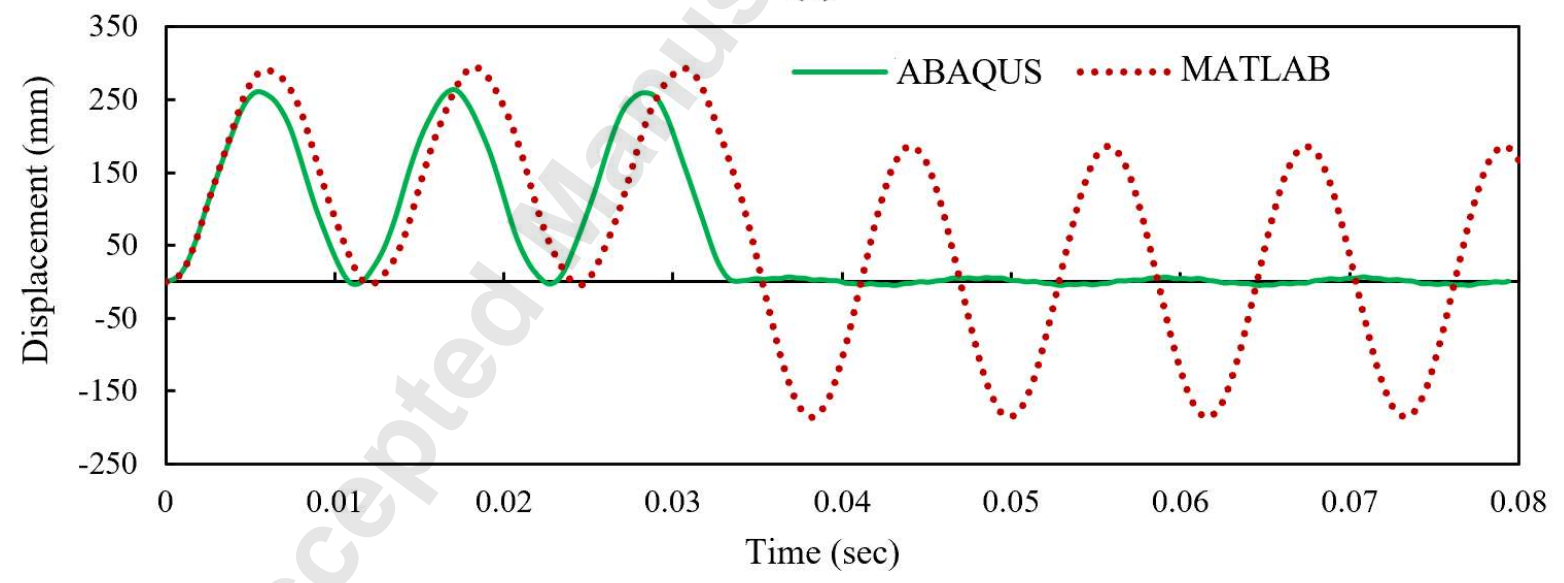

Figure 10. Correlation of displacement time histories between ABAQUS and the MATLAB code for (a) small, (b) medium, and (c) large deflections due to rectangular pulse load of magnitude $12 \mathrm{~N} / \mathrm{mm}$ and durations $2.2 \mathrm{msec}$ (impulsive), $10.1 \mathrm{msec}$ (dynamic) and $34 \mathrm{msec}$ (quasi-static). The full range of loading regimes is considered. 
(a)

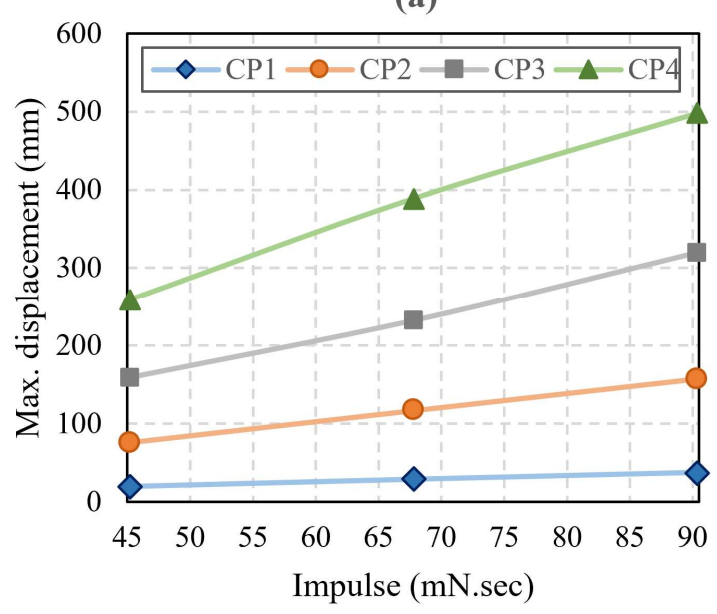

(b)

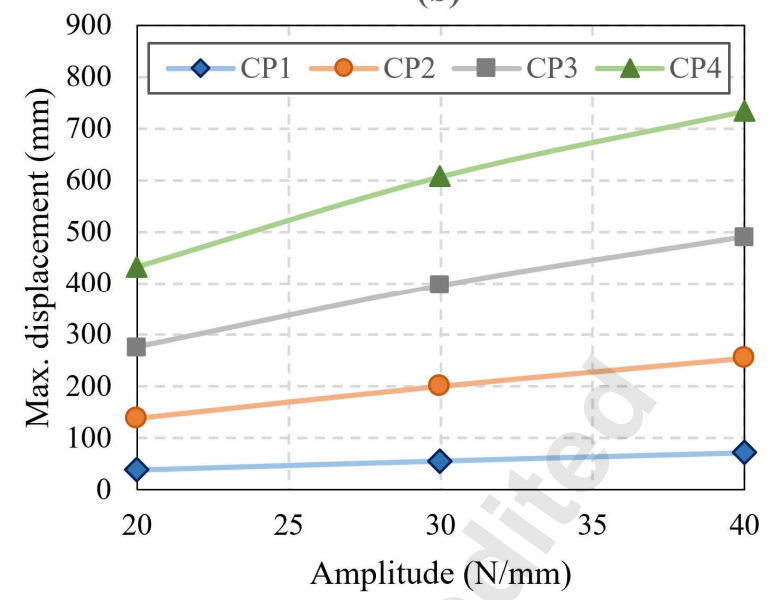

Fig. 11. Dependence of displacement on (a) impulse, and (b) pressure amplitude, for four control points equally spaced along the beam.

A final remark concerning simulation times is in order. While a direct comparison of the two models of the same mesh size is not possible due to the dimensionality difference between the two models (1D mathematical/MATLAB model and 2D ABAQUS model), it is possible to compare the simulation times for the converged models in each case. As far as linear perturbation analyses are concerned for a given natural mode the simulation time for MATLAB code is 90 seconds on a PC with a $3.5 \mathrm{GHz}$ core, which is comparable to the 40 seconds it takes to run a similar ABAQUS simulation. As for the dynamic response, it takes around 100-150 seconds to run the simple model in MATLAB, whereas it takes up to 8 minutes to run a similar simulation using $A B A Q U S / E x p l i c i t$. It is further found that introducing damping at the level assumed has minimal effect on the simulation time in each case.

\section{Conclusions}

In this study, the mathematical model of the non-prismatic Timoshenko beam considering shear and bending deformations is developed. The nonlinear equations of the beam dynamics are derived using the extended Hamilton's principle (Lagrange-D'Alembert's principle) under the condition of finite angles of rotation of the beam's neutral axis. The beam is also assumed to be made of an isotropic linear elastic material for which Hooke's law is valid.

The discrete model of the beam motion is evolved. It is based on the finite difference method that reduces partial differential equations to the system of ordinary differential equations of 
the first order. The PDEs, converted to ODEs are then numerically solved to derive the structural response and eigenfrequencies of the non-prismatic Timoshenko beam. The natural frequencies and vibration modes are computed for linear as well as for non-linear beam vibrations of the type expected in the problem. These results demonstrate decreasing the beam eigenfrequency with raising the amplitude of non-linear oscillations. It is obtained that the nonlinear mode shapes are homomorphic with those of linear modes.

The numerical analyses of forced vibrations of a given beam in the field of external pulse loading are carried out. The beam points are observed to oscillate in different manners depending on their relative positions along the beam. Points close to the free end of the beam are subject to almost harmonic oscillations, and the free end vibrates with a frequency equal to that of the external force. When a point approaches the clamped end of the beam, it oscillates in two-frequency mode and lags in phase from the oscillations of the free end. Some damping was introduced in this work to stabilize the response and the results of analyses at each stage were compared with those obtained by the commercial FE software, ABAQUS 2019.

The reliability of the developed numerical method was tested through the calculations of the dynamic reaction of a special two-dimensional Timoshenko beam subject to external pulse loads. Moderate and large amplitudes of pulse were selected and impulsive, dynamic, and quasi-static loading regimes, defined purely based on the ratio of loading duration to the natural period of the vibration, were considered. Under these circumstances, the evolution of the displacement of the free end of the beam calculated by the FDM was compared with similar data obtained by the commercial FE software, ABAQUS 2019. In all cases, a strong correlation exists between the two sets of results at the initial stage of the calculations, which is weakened as time elapses due to the fact that FDM does not take into account viscous damping. 


\section{References}

[1] Han, SM, Benaroya, H., \& Wei, T. (1999). Dynamics of transversely vibrating beams using four engineering theories. Journal of Sound and Vibration, 225 (5), 935-988.

[2] Kruszewski, ET (1949). Effect of transverse shear and rotary inertia on the natural frequency of a uniform beam, National Advisory Committee for Aeronautics (No. 1909). Technical Note.

[3] Mehreganian, N., Fallah, A. S., \& Sareh, P. (2021). Structural Mechanics of Negative Stiffness Honeycomb Metamaterials. Journal of Applied Mechanics, 88(5), 051006.

[4] Traill-Nash, R. W., \& Collar, A. R. (1953). The effects of shear flexibility and rotatory inertia on the bending vibrations of beams. The Quarterly Journal of Mechanics and Applied Mathematics, 6(2), 186-222.

[5] Wang, CM, Tan, VBC, \& Zhang, YY (2006). Timoshenko beam model for vibration analysis of multi-walled carbon nanotubes. Journal of Sound and Vibration, 294 (4-5), 1060-1072.

[6] Lee, S. Y., Ke, H. Y., \& Kuo, Y. H. (1990). Analysis of non-uniform beam vibration. Journal of Sound and Vibration, 142(1), 15-29.

[7] Ruta, P. (2004). The vibration of a non-prismatic beam on an inertial elastic half-plane. Journal of sound and vibration, 275 (3-5), 533-556.

[8] Wang, G.F. and X.Q. Feng, Timoshenko beam model for buckling and vibration of nanowires with surface effects. Journal of Physics D-Applied Physics, 2009. 42(15).

[9] Wang, C.M., V.B.C. Tan, and Y.Y. Zhang, Timoshenko beam model for vibration analysis of multi-walled carbon nanotubes. Journal of Sound and Vibration, 2006. 294(4-5): p. 1060-1072.

[10] Lin, S.C. and K.M. Hsiao, Vibration analysis of a rotating Timoshenko beam. Journal of Sound and Vibration, 2001. 240(2): p. 303-322.

[11] Rao, S.S. and R.S. Gupta, Finite element vibration analysis of rotating Timoshenko beams. Journal of Sound and Vibration, 2001. 242(1): p. 103-124.

[12] Yardimoglu, B., Vibration analysis of rotating tapered Timoshenko beams by a new finite element model. Shock and Vibration, 2006. 13(2): p. 117-126.

[13] Bazoune, A. and Y.A. Khulief, A Finite Beam Element for Vibration Analysis of Rotating Tapered Timoshenko Beams. Journal of Sound and Vibration, 1992. 156(1): p. 141-164.

[14] Karabalis, D.L. and D.E. Beskos, Static, Dynamic and Stability Analysis of Structures Composed of Tapered Beams. Computers \& Structures, 1983. 16(6): p. 731-748.

[15] Keller, T., T. Tirelli, and A.X. Zhou, Tensile fatigue performance of pultruded glass fiber reinforced polymer profiles. Composite Structures, 2005. 68(2): p. 235-245.

[16] Kuo, Y.H., T.H. Wu, and S.Y. Lee, Bending Vibrations of a Rotating Nonuniform Beam with Tip Mass and an Elastically Restrained Root. Computers \& Structures, 1992. 42(2): p. 229-236.

[17] Rossi, R.E. and P.A.A. Laura, Numerical Experiments on Vibrating, Linearly Tapered Timoshenko Beams. Journal of Sound and Vibration, 1993. 168(1): p. 179-183.

[18] Yardimoglu, B., A novel finite element model for vibration analysis of rotating tapered Timoshenko beam of equal strength. Finite Elements in Analysis and Design, 2010. 46(10): p. 838-842.

[19] Attarnejad, R., S.J. Semnani, and A. Shahba, Basic displacement functions for free vibration analysis of nonprismatic Timoshenko beams. Finite Elements in Analysis and Design, 2010. 46(10): p. 916-929.

[20] Attarnejad, R., A. Shahba, and M. Eslaminia, Dynamic basic displacement functions for free vibration analysis of tapered beams. Journal of Vibration and Control, 2011. 17(14): p. 2222-2238.

[21] Attarnejad, R., A. Shahba, and S.J. Semnani, Analysis of Non-Prismatic Timoshenko Beams Using Basic Displacement Functions. Advances in Structural Engineering, 2011. 14(2): p. 319-332.

[22] Shah, SKA, Fallah, AS, \& Louca, LA (2012). On the chaotic dynamic response of deterministic nonlinear single and multi-degree-of-freedom systems. Journal of mechanical science and technology, 26 (6), 1697-1709.

[23] Salarieh, H., \& Shahrokhi, M. (2007). Indirect adaptive control of discrete chaotic systems. Chaos, Solitons \& Fractals, 34(4), 1188-1201.

[24] Yan, Q. Y., Ding, H., \& Chen, L. Q. (2014). Periodic responses and chaotic behaviors of an axially accelerating viscoelastic Timoshenko beam. Nonlinear Dynamics, 78(2), 1577-1591.

[25] Sapountzakis, EJ, \& Kampitsis, AE (2010). Nonlinear dynamic analysis of Timoshenko beam-columns partially supported on tensionless Winkler foundation. Computers \& Structures, 88 (21-22), 1206-1219. 
[26] Shahlaei-Far, S., Nabarrete, A., \& Balthazar, JM (2016). Nonlinear vibrations of cantilever Timoshenko beams: a homotopy analysis. Latin American Journal of Solids and Structures, 13 (10), 1866-1877.

[27] Zhong, H., \& Liao, M. (2007). Higher-order nonlinear vibration analysis of Timoshenko beams by the splinebased differential quadrature method. Shock and Vibration, 14 (6), 407-416.

[28] Liao, M., \& Zhong, H. (2008). Nonlinear vibration analysis of tapered Timoshenko beams. Chaos, Solitons \& Fractals, 36(5), 1267-1272.

[29] Torabi, K., Sharifi, D., \& Ghassabi, M. (2017). Nonlinear vibration analysis of a Timoshenko beam with concentrated mass using variational iteration method. Journal of the Brazilian Society of Mechanical Sciences and Engineering, 39(12), 4887-4894.

[30] Ruta, P., \& Szybiński, J. (2015). Nonlinear analysis of nonprismatic Timoshenko beam for different geometric nonlinearity models. International Journal of Mechanical Sciences, 101, 349-362.

[31] Palacios, R. (2011). Nonlinear normal modes in an intrinsic theory of anisotropic beams. Journal of Sound and Vibration, 330(8), 1772-1792.

[32] Radgolchin, M., \& Moeenfard, H. (2018). An analytical approach for modeling nonlinear vibration of doubly clamped functionally graded Timoshenko microbeams using strain gradient theory. International Journal of Dynamics and Control, 6(3), 990-1007.

[33] Chakrabarti, A., Ray, P. C., \& Bera, R. K. (2010). Large amplitude free vibration of a rotating nonhomogeneous beam with nonlinear spring and mass system. Journal of vibration and acoustics, 132(5).

[34] Şimşek, M. (2016). Nonlinear free vibration of a functionally graded nanobeam using nonlocal strain gradient theory and a novel Hamiltonian approach. International Journal of Engineering Science, 105, 12-27.

[35] Shang-Rou, H., Shaw, S. W., \& Pierre, C. (1994). Normal modes for large amplitude vibration of a cantilever beam. International Journal of Solids and Structures, 31(14), 1981-2014.

[36] Awrejcewicz J., Krysko V., Soldatov V., Krysko V.A. (2012) Analysis of non-linear dynamics of the Timoshenko flexible beams using wavelets. Transactions of the ASME, 7,011005-1-011005-14

[37] Hibbit, H. D., Karlsson, B. I., \& Sorensen, E. P. (2016). ABAQUS user manual, version 6.12. Simulia, Providence, RI.

[38] Weaver Jr, W., Timoshenko, S. P., \& Young, D. H. (1990). Vibration problems in engineering. John Wiley \& Sons. 